\title{
Nonadiabatic Transition in the A-Band Photodissociation of Ethyl Iodide from 294 to 308 nm by Using Velocity Imaging Detection
}

\author{
Xiao-Peng Zhang, Wei-Bin Lee, and King-Chuen Lin* \\ Department of Chemistry, National Taiwan University, Taipei 106, and Institute of Atomic and Molecular \\ Sciences, Academia Sinica, Taipei 106, Taiwan
}

Received: June 11, 2008; Revised Manuscript Received: July 21, 2008

\begin{abstract}
Photodissociation dynamics of ethyl iodide in the A-band is investigated at wavelengths between 294 and $308 \mathrm{~nm}$ using resonance-enhanced multiphoton ionization technique combined with velocity imaging detection. The I/I* branching, translational energy disposals, anisotropy parameters, and curve crossing probabilities of the dissociation channels are determined. The $\mathrm{I}\left(5 \mathrm{p}^{2} \mathrm{P}_{3 / 2}\right)$-product channel is found to have hotter internal states of $\mathrm{C}_{2} \mathrm{H}_{5}$, and the $\mathrm{I}^{*}\left(5 \mathrm{p}^{2} \mathrm{P}_{1 / 2}\right)$ channel is accompanied by colder $\mathrm{C}_{2} \mathrm{H}_{5}$. Anisotropy parameters $(\beta)$ for the I* channel remain at 2.0, indicating that the $\mathrm{I}^{*}$ production should originate from the ${ }^{3} \mathrm{Q}_{0}$ state. The $\beta$ values are from 1.6 to 1.9 in the I-product channel, which comprises two components of direct excitation of ${ }^{3} \mathrm{Q}_{1}$ and nonadiabatic transition between the ${ }^{3} \mathrm{Q}_{0}$ and ${ }^{1} \mathrm{Q}_{1}$ states. The curve crossing probability rises rapidly around the conical intersection but remains almost constant after passing through the curve crossing. The ${ }^{1} \mathrm{Q}_{1}$ and ${ }^{3} \mathrm{Q}_{0}$ states in the exit region are thus expected to cross almost parallel along the dissociation coordinate. As compared to the case of $\mathrm{CH}_{3} \mathrm{I}$, the nonadiabatic transition probabilities are slightly enhanced by an ethylsubstituted group.
\end{abstract}

\section{Introduction}

Photochemistry of alkyl halides has received wide attention for decades, in part because of their importance to the environmental impact. Alkyl halides may undergo $\mathrm{C}-\mathrm{X}(\mathrm{X}=$ $\mathrm{Cl}, \mathrm{Br}, \mathrm{I}$ ) bond fission, upon UV excitation to the A-band (n, $\left.\sigma^{*}\right)$ that comprises three overlapping states denoted as ${ }^{3} \mathrm{Q}_{1},{ }^{3} \mathrm{Q}_{0}$, and ${ }^{1} \mathrm{Q}_{1}$ in the ascending order by Mulliken. ${ }^{1}$ The A-band photodissociation of $\mathrm{CH}_{3} \mathrm{I}$ leads to $\mathrm{CH}_{3}$ and spin-orbit ground $\mathrm{I}\left(5 \mathrm{p}{ }^{2} \mathrm{P}_{3 / 2}\right)$ or spin- orbit excited $\mathrm{I}^{*}\left(5 \mathrm{p}{ }^{2} \mathrm{P}_{1 / 2}\right) .{ }^{2-13}$ The ${ }^{3} \mathrm{Q}_{0}\left(2 \mathrm{~A}_{1}\right)$ state correlates to the I* fragment with a transition dipole moment aligned parallel to the $\mathrm{C}-\mathrm{I}$ bond, whereas the ${ }^{3} \mathrm{Q}_{1}(2 \mathrm{E})$ and ${ }^{1} \mathrm{Q}_{1}(3 \mathrm{E})$ states correlate to I with the transition dipole moment perpendicular to the bond axis. I* was reported as the major product, and a small I product was attributed to a curve crossing from the ${ }^{3} \mathrm{Q}_{0}\left(2 \mathrm{~A}_{1}\right)$ to ${ }^{1} \mathrm{Q}_{1}(3 \mathrm{E})$ state. $\mathrm{CH}_{3} \mathrm{I}$ may evolve to a $C_{s}$ symmetry during dissociation, when a $\mathrm{H}_{3} \mathrm{C}-\mathrm{I}$ bending mode is coupled to the dissociation axis. ${ }^{14-16}$ The ${ }^{1} \mathrm{Q}_{1}(3 \mathrm{E})$ state therefore splits into $\mathrm{A}^{\prime}$ and $\mathrm{A}^{\prime \prime}$ states, and the coupling between the ${ }^{3} \mathrm{Q}_{0}\left(3 \mathrm{~A}^{\prime}\right)$ and ${ }^{1} \mathrm{Q}_{1}\left(4 \mathrm{~A}^{\prime}\right)$ states enhances the nonadiabatic transition. Such nonadiabatic nature of the multidimensional photodissociation has strong influence on the ultrafast reaction rates for each dissociation channel. ${ }^{17}$ The resulting $\mathrm{CH}_{3}$ moiety is thus complicated with various multimode excitation but dominated by the umbrella mode. ${ }^{6}$

As compared with methyl iodide, the photodissociation dynamics of $\mathrm{C}_{2} \mathrm{H}_{5} \mathrm{I}$ and other $\alpha$-branched heavier alkyl iodides have been less studied. In general, these alkyl iodides dissociate as rapidly as $\mathrm{CH}_{3} \mathrm{I}$ in the regime of $10-200 \mathrm{fs}$. Such an ultrafast dynamic behavior has been characterized in terms of resonance Raman or photoelectron spectra from the alkyl iodide cations as measured in gaseous vapors or nonpolar solvents. ${ }^{6,18-20}$ Despite rapid dissociation, the $\alpha$-branched alkyl iodides having lower molecular symmetry should cause difference in the

* Author to whom correspondence should be addressed. Email: kclin@ ccms.ntu.edu.tw. Fax: 886-2-23621483. nonadiabatic transition. According to the one-dimensional Landau-Zener model, the heavier branches lead to a slower velocity through the curve crossing region such that the ${ }^{3} \mathrm{Q}_{0}$ state may have more probability to transit into the ${ }^{1} \mathrm{Q}_{1}$ state. ${ }^{21}$ For instance, $\Phi\left(I^{*}\right)$, a quantum yield of $I^{*}$, for $\mathrm{CH}_{3} \mathrm{I}$ was obtained as about $0.73 \pm 0.04$ in the wavelength range $248-266$ $\mathrm{nm}$, and then decreased to $0.13 \pm 0.07$ at $304 \mathrm{~nm} .{ }^{12,22}$ When a bulkier size is branched to the $\alpha$-carbon atom, $\Phi\left(I^{*}\right)$ was obtained to be $0.44 \pm 0.03$ and $0.20 \pm 0.03$ for isopropyl and isobutyl iodide, respectively, at $266 \mathrm{~nm},{ }^{23}$ and 0.03 for tertbutyl iodide at $277 \mathrm{~nm} .{ }^{24}$ The $\alpha$-bulky group enhances the strength of the ${ }^{3} \mathrm{Q}_{0} \rightarrow{ }^{1} \mathrm{Q}_{1}$ nonadiabatic transition and brings about the decrease of $\Phi\left(I^{*}\right)$. Although a qualitative agreement is reached in their dynamic trend, controversy still exists in understanding the detailed complexity..$^{25,26}$

As compared to $\mathrm{CH}_{3} \mathrm{I}$, the A-band absorption spectrum of $\mathrm{C}_{2} \mathrm{H}_{5} \mathrm{I}$ appears to slightly blue shift to a region of 200-310 $\mathrm{nm}$, peaking at $258 \mathrm{~nm} \cdot{ }^{27,28}$ Energy partitioning and $\mathrm{I} * / \mathrm{I}$ branching have been the focus of the study. $21,25,29,30$ The consequences rely on the excitation wavelength and the nonadiabatic nature of the A-band photodissociation. A recent study of the photodissociation of ethyl iodide over the wavelength range of $245-283 \mathrm{~nm}$ provided the quantum yields of I and I* as well as the related anisotropy parameters. ${ }^{31}$ Accordingly, the curve crossing probabilities were determined to rise gradually with the wavelength. But the photolyzing wavelengths are away from the region of the conical intersection such that the behavior of the nonadiabatic transition can not be effectively compared with the case of $\mathrm{CH}_{3} \mathrm{I}$. By taking advantage of resonanceenhanced multiphoton ionization (REMPI) technique combined with velocity imaging detection, we extend the photolyzing wavelengths to $294-308 \mathrm{~nm}$ in this work, thereby looking into the influence of geometric difference on the photodissociation dynamics of $\mathrm{C}_{2} \mathrm{H}_{5} \mathrm{I}$. The speed and angular distributions of I and $\mathrm{I}^{*}$ are analyzed to determine the subsequent spatial anisotropies and the relative quantum yields at different pho- 
tolyzing wavelengths. The curve crossing probabilities between the ${ }^{3} \mathrm{Q}_{0}$ and ${ }^{1} \mathrm{Q}_{1}$ states are then evaluated and compared to the case of methyl iodide to understand the ethyl-substituted effect.

\section{Experimental Section}

The apparatus is similar to that used previously. ${ }^{31-33} \mathrm{~A}<5 \%$ $\mathrm{C}_{2} \mathrm{H}_{5} \mathrm{I}$ sample (99\% purity) was carried by helium gas at $1 \mathrm{~atm}$ through a pulsed valve with $0.6 \mathrm{~mm}$ diameter orifice (General Valve Co.), operating at $10 \mathrm{~Hz}$ and expanded into the source chamber. After passing through a $1 \mathrm{~mm}$ diameter skimmer and a collimator, the molecular beam was intersected perpendicularly by a linearly polarized laser beam in a two-stage ion lens region. The skimmer was mounted $30 \mathrm{~mm}$ downstream from the nozzle to divide the source chamber from the detection chamber.

A $308 \mathrm{~nm}$ excimer laser-pumped dye laser (FL3002, Lambda Physik) was frequency-doubled to emit the wavelengths from 294 to $308 \mathrm{~nm}$ with the energy $20-200 \mu \mathrm{J}$, followed by linear polarization perpendicular to the flight tube direction, and then focused at the skimmed beam with a $200 \mathrm{~mm}$ focal-length lens. The laser pulses were focused to the leading edge of the molecular beam to minimize the cluster formation. The $\mathrm{C}_{2} \mathrm{H}_{5} \mathrm{I}$ molecules were then photolyzed and the subsequent I and I* fragments, as selectively ionized by a $(2+1)$ REMPI scheme, were velocity-mapped onto a two-stage microchannel plate (MCP) and a phosphor screen (FM3040, Galileo). The MCP could be gated within a minimum duration of $250 \mathrm{~ns}$ for mass selection. The ion imaging on the phosphor screen was recorded by a charge coupled device (CCD) camera (200XL4078, Pixelfly). The laser wavelength was scanned back and forth within the range of Doppler broadening to cover all the velocity components of the selected fragments. All the ion signals without the gate restriction may be transferred to a transient digitizer for display of the mass spectrum. The laser power dependence of the ion intensity was acquired directly from the transient digitizer.

\section{Results and Discussion}

A. I and I* Ion Images. The ion images of I and $I^{*}$ photofragments were acquired at four pairs of dissociation wavelengths, 294.1/298.2, 303.7/304.0, 304.7/305.6, 306.6/307.6 $\mathrm{nm}$, beyond which no I/I* pairs can be probed significantly in the A-band absorption. Each raw image was accumulated over 40000 laser shots with the background removed and then restored to its Abel-inversed three-dimensional spatial distribution of the fragment. Examples of the raw and reconstructed ion images are given in Figures 1 and 2. The corresponding speed distributions of $\mathrm{I}^{*}$ and I extracted from these ion images are also shown in the figures. Each of them can be well fitted by a narrow Gaussian curve, suggesting that I and $\mathrm{I}^{*}$ are produced from direct dissociation along the repulsive potential energy surfaces after absorbing one UV photon.

The $\mathrm{I}^{+}$contribution alternatively by the dissociative ionization channels should be negligible. They may be expressed by

$$
\begin{gathered}
\mathrm{C}_{2} \mathrm{H}_{5} \mathrm{I}+2 h v \rightarrow \mathrm{C}_{2} \mathrm{H}_{5} \mathrm{I}^{+} \\
\mathrm{C}_{2} \mathrm{H}_{5} \mathrm{I}^{+}+h v \rightarrow \mathrm{C}_{2} \mathrm{H}_{5}+\mathrm{I}^{+} \\
\mathrm{C}_{2} \mathrm{H}_{5} \mathrm{I}^{+} \rightarrow \mathrm{C}_{2} \mathrm{H}_{5}{ }^{+}+\mathrm{I} \\
\mathrm{I}+3 h v \rightarrow \mathrm{I}^{+}
\end{gathered}
$$

The channels via eqs 1 and 2 are blocked as a result of the wavelength larger than $266 \mathrm{~nm}$. The details were discussed previously. ${ }^{31}$ If the dissociative ionization channel proceeds
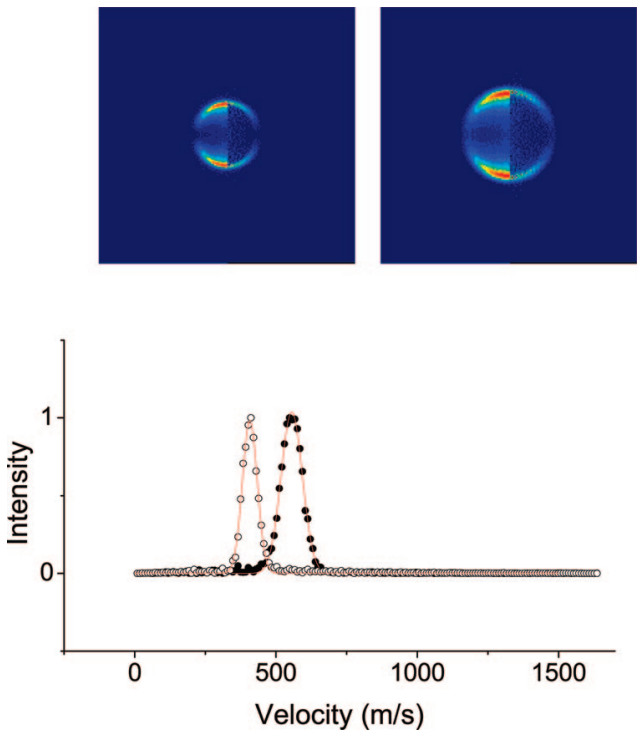

Figure 1. Ion images and speed distributions of I (right) and I* (left) from the photodissociation of $\mathrm{C}_{2} \mathrm{H}_{5} \mathrm{I}$ at 303.7 and $304.0 \mathrm{~nm}$. The left half is the raw ion image, and the right half is the inverse Abeltransformed one. The laser beam is linearly polarized along the vertical direction. The normalized I and I* speed distributions are denoted by the closed-circle and open-circle data points, respectively. The Gaussian fits are denoted by the solid lines.
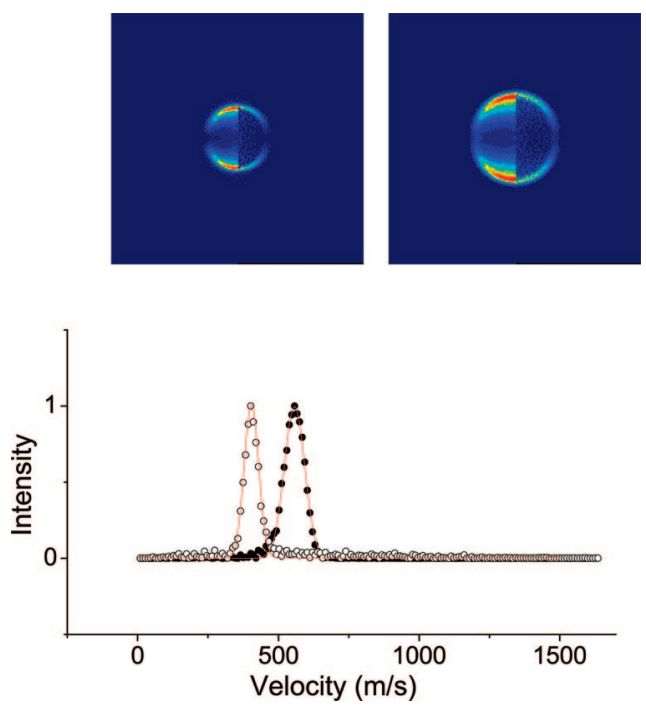

Figure 2. Ion images and speed distributions of I (right) and I* (left) from the photodissociation of $\mathrm{C}_{2} \mathrm{H}_{5} \mathrm{I}$ at 304.7 and $305.6 \mathrm{~nm}$. The left half is the raw ion image, and the right half is the inverse Abeltransformed one. The laser beam is linearly polarized along the vertical direction. The normalized I and I* speed distributions are denoted by the closed-circle and open-circle data points, respectively. The Gaussian fits are denoted by the solid lines.

through eqs 3 and 4, then the achievement requires five photons. The proposed mechanism is against the measurements of laser power dependence yielding a slope about 3 , which implies that the ionization step of iodine is saturated.

The available energy $E_{\text {avl }}$ for the dissociation process may be calculated by

$$
E_{\mathrm{avl}}=E_{h v}-D_{0}-E_{\mathrm{el}}+E_{\mathrm{int}}
$$

where $E_{h v}$ is the photon energy; $D_{0}$ is the $\mathrm{C}-\mathrm{I}$ bond dissociation energy evaluated to be $2.353 \mathrm{eV}$ for $\mathrm{C}_{2} \mathrm{H}_{5} \mathrm{I} ;{ }^{34} E_{\mathrm{el}}$ is the electronic energy level of the iodine atom, zero for I and $7603 \mathrm{~cm}^{-1}$ for I*; and $E_{\text {int }}$ is the internal energy of the parent molecule, which 
TABLE 1: Summary of Dynamical Parameters in the Photodissociation of $\mathrm{C}_{2} \mathrm{H}_{5} \mathrm{I}$

\begin{tabular}{|c|c|c|c|c|c|c|c|}
\hline $\begin{array}{l}\text { wavelength } \\
\quad(\mathrm{nm})\end{array}$ & state & $\begin{array}{c}E_{\mathrm{avl}} \\
(\mathrm{kJ} / \mathrm{mol})\end{array}$ & $\begin{array}{c}E_{\mathrm{t}} \\
(\mathrm{kJ} / \mathrm{mol})\end{array}$ & $f_{\mathrm{t}}$ & $\beta$ & $\Phi\left(I^{*}\right)$ & $P_{\mathrm{cc}}$ \\
\hline 294.1 & $I^{*}$ & 88.6 & 65.6 & 0.74 & 2.0 & $0.51 \pm 0.10$ & $0.45 \pm 0.10$ \\
\hline 298.2 & I & 173.9 & 111.6 & 0.64 & $1.6 \pm 0.1$ & & \\
\hline 304.0 & $I^{*}$ & 75.2 & 56.8 & 0.76 & 2.0 & 0.20 & 0.81 \\
\hline 303.7 & I & 166.4 & 107 & 0.64 & $1.7 \pm 0.1$ & & \\
\hline 305.6 & $I^{*}$ & 73.6 & 55.5 & 0.75 & 2.0 & 0.20 & 0.78 \\
\hline 304.7 & I & 165.1 & 105.3 & 0.64 & $1.7 \pm 0.1$ & & \\
\hline 306.6 & $I^{*}$ & 72.1 & 51.2 & 0.71 & 2.0 & 0.21 & 0.79 \\
\hline 307.6 & I & 161.5 & 96.5 & 0.61 & $1.9 \pm 0.1$ & & \\
\hline
\end{tabular}

is considered as zero in a supersonic molecular beam. The center-of-mass translational energy carried by the dissociating species can be evaluated from the velocity of an individual fragment. The fraction of the translational energy release, $f_{\mathrm{T}}$, defined as a ratio of the average translational energy $\left\langle E_{\mathrm{T}}\right\rangle$ to the available energy $E_{\mathrm{avl}}$, is then estimated to be $0.61-0.64$ and $0.71-0.76$ for the I and $I^{*}$ channels, respectively, indicating that the I channel may carry more internal energy in the $\mathrm{C}_{2} \mathrm{H}_{5}$ moiety. This fact is also reflected in a wider speed distribution of the I fragment (Figures 1 and 2), which suggests that the accompanied $\mathrm{C}_{2} \mathrm{H}_{5}$ be in hotter internal states than the other channel leading to $\mathrm{I}^{*}+\mathrm{C}_{2} \mathrm{H}_{5}$. The $f_{\mathrm{T}}(\mathrm{I})$ and $f_{\mathrm{T}}\left(\mathrm{I}^{*}\right)$ values are consistent with 0.68 and 0.72 , respectively, obtained at $304 \mathrm{~nm},{ }^{25}$ and comparable with $0.62-0.65$ and $0.72-0.75$ obtained at the shorter photolyzing wavelengths from 245 to $283 \mathrm{~nm} .^{21,31}$ They are also comparable with those obtained in $\mathrm{CH}_{3} \mathrm{I}$. But $f_{\mathrm{T}}(\mathrm{I})$ and $f_{\mathrm{T}}\left(\mathrm{I}^{*}\right)$ may be reduced to $0.52-0.55$ and $0.55-0.59$, respectively, at either 266 or $304 \mathrm{~nm}$ in $\mathrm{ICH}_{2} \mathrm{CN}$ in which an electronwithdrawing group is substituted. ${ }^{35}$ More energy is deposited in the internal states of $\mathrm{CH}_{2} \mathrm{CN}$.

The relative quantum yields $\Phi$ of the I and I* channels may be evaluated by

$$
\Phi\left(\mathrm{I}^{*}\right)=\frac{N\left(\mathrm{I}^{*}\right)}{N(\mathrm{I})+N\left(\mathrm{I}^{*}\right)}=k \frac{S\left(\mathrm{I}^{*}\right)}{S(\mathrm{I})+S\left(\mathrm{I}^{*}\right)}
$$

and

$$
\Phi(\mathrm{I})=1-\Phi\left(\mathrm{I}^{*}\right)
$$

Here, $N\left(\mathrm{I}^{*}\right)$ and $N(\mathrm{I})$ are the numbers of $\mathrm{I}^{*}$ and I fragments; $S\left(\mathrm{I}^{*}\right)$ and $S(\mathrm{I})$ are the REMPI signals that are measured individually. The factor $k$, which relates the obtained iodine ion signal to its state population, can be evaluated by performing a calibration experiment of $\mathrm{I}_{2}$ photolysis under the same conditions. The factor $k$ was determined to be $5.9,0.25,2.0$, and 0.67 at the photolyzing wavelengths of 294.05/298.23, 303.69/ $304.03,304.68 / 305.57$, and 306.63/307.64 nm, respectively, by assuming identical intensities of the $\mathrm{I}$ and $\mathrm{I}^{*}$ fragments at each pair of wavelengths. The $\Phi\left(I^{*}\right)$ value thus obtained decreases from 0.51 to 0.2 with increasing the photolyzing wavelength, and then remains almost at 0.2 . Their results are listed in Table 1.

In a photodissociation process, angular distribution $I(\theta)$ of the fragment is obtained by integrating the reconstructed threedimensional spatial distribution over a proper range of speed at each angle. It may be characterized by an anisotropy parameter, $\beta$, according to the following equation, ${ }^{36,37}$

$$
I(\theta)=(4 \pi)^{-1}\left[1+\beta P_{2}(\cos \theta)\right]
$$

Here, $\mathrm{P}_{2}(\cos \theta)$ is the second-order Legendre polynomial, and $\theta$ is the angle between the laser polarization direction and the recoil velocity of fragments. The $\beta$ value can be determined by least-squares fit to the angular distribution. Figure 3 gives an example for the $\beta(\mathrm{I})$ and $\beta\left(\mathrm{I}^{*}\right)$ fits at the wavelengths of $303.7 /$ $304.0 \mathrm{~nm}$. In this manner, the $\beta$ values are obtained at various wavelengths. The dynamical properties at the wavelengths studied are all listed in Table 1. It should be noted that the obtained anisotropy parameters may be somehow interfered with by the laser-induced molecular alignment effect, when a single laser radiation is employed for the role of both photolysis and ionization. Dynamic alignment of $\mathrm{CH}_{3} \mathrm{I}$ was previously reported by nanosecond laser pulses at intensities $<10^{12} \mathrm{~W} / \mathrm{cm}^{2}$ and by picosecond laser pulses at higher intensities of $10^{14}-10^{15}$ $\mathrm{W} / \mathrm{cm}^{2}{ }^{38-40}$ The use of a laser field of moderate intensity can result in molecular alignment due to the coupling of the laser field with the induced dipole moment. Once it happens, the angular distribution of subsequent atomic fragments may be characterized by a product of the orientational probability distribution of the parent molecule and the recoil distribution given in eq 8 for the fast axial photodissociation. ${ }^{41}$ In this work, the maximum energy intensity is controlled as small as $<5 \times$ $10^{9} \mathrm{~W} / \mathrm{cm}^{2}$. Thus, the caused molecular and atomic alignment effect should be minimized.

B. Nonadiabatic Transition. The A-band of ethyl iodide is mainly contributed by ${ }^{3} \mathrm{Q}_{0}$ covering $81 \%$ of the total absorption cross section with the maximum at $38820 \mathrm{~cm}^{-1}$ and full width at half-maximum (fwhm) of $5060 \mathrm{~cm}^{-1}$. The other two small components are ${ }^{3} \mathrm{Q}_{1}$ sharing $9 \%$ with the maximum at 36500 $\mathrm{cm}^{-1}$ and fwhm $4060 \mathrm{~cm}^{-1}$, and ${ }^{1} \mathrm{Q}_{1}$ sharing $10 \%$ with the maximum at $40275 \mathrm{~cm}^{-1}$ and fwhm $3530 \mathrm{~cm}^{-1}$. 42 Thus, the ${ }^{3} \mathrm{Q}_{0}$ and ${ }^{3} \mathrm{Q}_{1}$ states dominate over the excitation wavelengths studied. The $\beta\left(\mathrm{I}^{*}\right)$ values over the full wavelengths are obtained to be 2.0 (Table 1), suggesting that the I* product should be contributed from the ${ }^{3} \mathrm{Q}_{0}$ state alone. Apart from the direct excitation of ${ }^{3} \mathrm{Q}_{1}$, the I channel with a positive $\beta(\mathrm{I})$ indicates

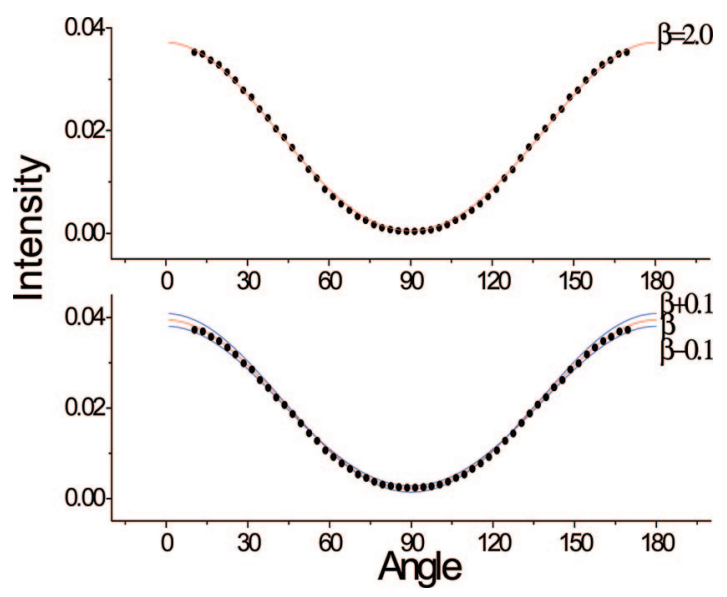

Figure 3. $\beta$ determination by the fit of angular distribution in the photodissociation of $\mathrm{C}_{2} \mathrm{H}_{5} \mathrm{I}$. (upper) $\beta=2.0$ at $304.0 \mathrm{~nm}$ for the $\mathrm{I}^{*}$ product channel, and (lower) $\beta=1.7 \pm 0.1$ at $303.7 \mathrm{~nm}$ for the I-product channel. 

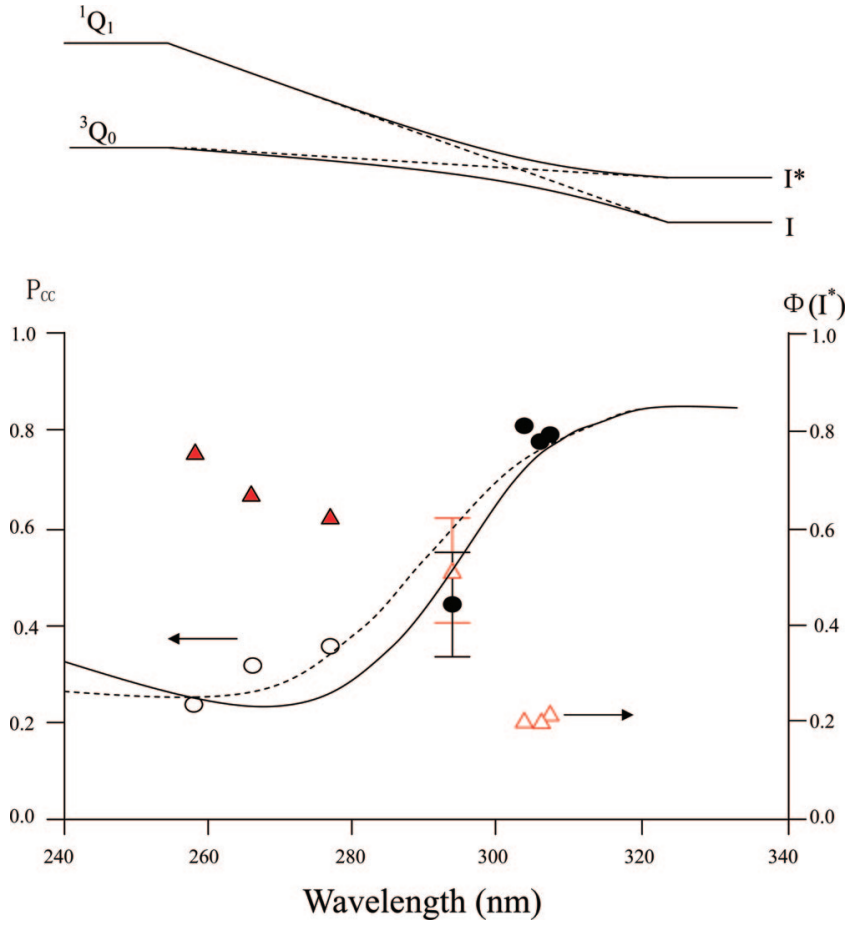

Figure 4. Nonadiabatic transition probability and quantum yield of the $I^{*}$ channel as a function of the dissociation wavelength. $(O)$ and $(\Delta)$ denote the data adopted from ref 31 , and $(\bullet)$ and $(\Delta)$ denote the present work. The solid curve is from the experimental findings in $\mathrm{CH}_{3} \mathrm{I}$ (ref 12), and the dashed line is expected by the Landau-Zener model.

that a significant contribution comes from the ${ }^{3} \mathrm{Q}_{0} \rightarrow{ }^{1} \mathrm{Q}_{1}$ nonadiabatic transition. The direct contribution in the I channel is considered to have a perpendicular character $(\beta=-1)$, whereas the nonadiabatic contribution remains the same anisotropy as the $\mathrm{I}^{*}$ channel. Therefore, the $\beta(\mathrm{I})$ value may be resolved into the relative contributions of the parallel and perpendicular components by the formula,

$$
\beta(I)=a \beta_{\|}+b \beta_{\perp}=a \beta\left(I^{*}\right)+b(-1)
$$

where $a$ and $b$, having a sum equal to unity, denote the fractions of nonadiabatic and direct-excitation contributions in the Iproduct channel. The curve crossing probability, $P_{\mathrm{cc}}$, between the ${ }^{3} \mathrm{Q}_{0}$ and ${ }^{1} \mathrm{Q}_{1}$ states can be evaluated by the following equation, ${ }^{12,31}$

$$
P_{\mathrm{cc}}=\frac{a\left(1-\Phi\left(\mathrm{I}^{*}\right)\right)}{\Phi(\mathrm{I} *)+a(1-\Phi(\mathrm{I} *))}
$$

Given the measures of $\beta(\mathrm{I}), \beta\left(\mathrm{I}^{*}\right)$, and $\Phi\left(\mathrm{I}^{*}\right), P_{\mathrm{cc}}$ may be determined to be $0.45 \pm 0.10,0.81,0.78$, and 0.79 at 294.05/ 298.23, 303.69/304.03, 304.68/305.57, 306.63/307.64 nm, respectively. The results are also listed in Table 1.

Figure 4 shows the nonadiabatic transition probability as a function of the excitation wavelength from 258 to $308 \mathrm{~nm}$, in comparison with those obtained in the $\mathrm{CH}_{3}$ I photodissociation. ${ }^{12}$ The data at $<280 \mathrm{~nm}$ are referred to the previous work. ${ }^{31}$ The wavelength dependence of $\Phi\left(\mathrm{I}^{*}\right)$ is also shown in Figure 4. Note that the datum at 294.05/298.23 nm carries a large uncertainty, because the selected wavelengths in this pair are far from each other. In the calibration experiments in which $\mathrm{I}_{2}$ is dissociated individually at 294.1 and $298.2 \mathrm{~nm}$, we neglect the wavelength dependence of photodissociation cross section of $\mathrm{I}_{2}$. That is, the resulting $\mathrm{I}$ and $\mathrm{I}^{*}$ intensities are assumed to be the same at these two wavelengths in determining $\Phi\left(I^{*}\right)$ and the subsequent $P_{\mathrm{cc}}$. However, this assumption is skeptical at such far separated wavelengths and the datum obtained at $294.05 / 298.23 \mathrm{~nm}$ is less reliable.

In the photodissociation of $\mathrm{CH}_{3} \mathrm{I}$, the location of the ${ }^{3} \mathrm{Q}_{0}-{ }^{1} \mathrm{Q}_{1}$ curve crossing has been predicted experimentally and theoretically. As photodissociation occurs in a closer distance to the Landau-Zener type crossing point, the nonadiabatic transition probability is expected to increase. On the basis of a sharp increase in the perpendicular component for the emission spectra, Butler and co-workers suggested that the curve crossing should lie at $2.35 \AA$ along the $\mathrm{C}$ - I bond coordinate with the excitation wavelength between 298.5 and $303.0 \mathrm{~nm},{ }^{43}$ which agrees with the calculated result of $298.2 \mathrm{~nm}$ by Alekseyev et al., ${ }^{16}$ but different from the prediction of $324.2 \mathrm{~nm}$ by Ajitha et al. ${ }^{44}$ Alekseyev et al. recently obtained the ${ }^{3} \mathrm{Q}_{0}-{ }^{1} \mathrm{Q}_{1}$ crossing at the C-I distance of $2.37 \AA$ and the umbrella angle of $114^{\circ}$ in the calculations of two-dimensional potential energy surfaces. ${ }^{16}$ The results are consistent with the previous theoretical predictions of $2.38 \AA$ and $114^{\circ}$ as well as $2.34 \AA$ and $114.3^{\circ} .14,44$

The curve crossing may be alternatively estimated by evoking a modified one-dimensional Landau-Zener theory as expressed by 22,45

$$
\Phi\left(\mathrm{I}^{*}\right)=\exp \left(-\frac{\rho}{2\left(E_{h v}-E_{\mathrm{cc}}\right)^{1 / 2}}\right)
$$

where $\rho$ denotes the modified Landau-Zener parameter which remains constant for the same system, $E_{h v}$ is the photodissociation energy, and $E_{\mathrm{cc}}$ is the energy of the curve crossing point. Given the quantum yields of $\mathrm{I}^{*}$ at different photodissociation energies, $E_{\mathrm{cc}}$ may be readily estimated. In terms of $\Phi\left(\mathrm{I}^{*}\right)$ obtained at 266 and $304 \mathrm{~nm}$, Li et al. ${ }^{22}$ estimated the potential energy at the curve crossing point to be $32790 \mathrm{~cm}^{-1}$, equivalent to an excitation wavelength of $305.0 \mathrm{~nm}$ which is slightly longer than those obtained above. ${ }^{16,43}$

Our results in Figure 4 reveal that the nonadiabatic transition probability increases gradually in the early stage and then rises up dramatically with the increased wavelength. The abrupt increase of the perpendicular character suggests that the conical intersection between the ${ }^{3} \mathrm{Q}_{0}$ and ${ }^{1} \mathrm{Q}_{1}$ states may be around 304 $\mathrm{nm} .{ }^{43}$ Given four $\Phi\left(\mathrm{I}^{*}\right)$ data obtained at 266.5/266.6, 277.4/ $277.9,303.7 / 304.0$, and $304.7 / 305.6 \mathrm{~nm}$ that are substituted in eq 11 , the energy at the curve crossing point is estimated to be $32602-32475 \mathrm{~cm}^{-1}$, corresponding to the excitation wavelength of 306.7-307.9 $\mathrm{nm}$. The reliable curve crossing point is thus considered to be between 304 and $306.7 \mathrm{~nm}$. When the dissociation wavelength is tuned to pass the conical intersection, $P_{\text {cc }}$ drops only by $<4 \%$, suggesting that these two curves may cross almost parallel in the exit channel. Unfortunately, no significant signals of the I/I* pairs may be obtained when the photolyzing wavelength exceeds $308 \mathrm{~nm}$. On the other hand, $\Phi\left(I^{*}\right)$ decreases with the wavelength and then rises slightly after passing the conical intersection. Increasing the ${ }^{3} \mathrm{Q}_{0} \rightarrow{ }^{1} \mathrm{Q}_{1}$ nonadiabatic transition should bring about the decrease of $\Phi\left(I^{*}\right)$. As shown in Figure 4, the nonadiabatic transition probabilities in this work behave similarly as those observed in the case of $\mathrm{CH}_{3} \mathrm{I}$ but are slightly enhanced by the branched ethyl group at a given photolyzing wavelength. The $P_{\mathrm{cc}}$ value is expected to increase as the $\alpha$-branched alkyl group becomes massive and the subsequent moving speed through the Landau-Zener crossing region becomes slower.

The vibrational spectra of the alkyl iodide cations are dominated by a long progression of CI stretch mode, followed by a CCI (or HCI in methyl iodide) bending mode (fundamental and combination). ${ }^{6,18-20,46}$ These two modes are associated with 
the earlier dissociation behavior and disappear after breaking apart of the cations. The photodissociation process is thus anticipated to involve coupling primarily between the CI internal coordinate and the bending mode. The nonadiabatic transition is substantially affected by the coupling strength between crossing curves. The one-dimensional Landau-Zener theory indicates that a stronger coupling between two crossing states may result in a larger adiabatic curve splitting and the subsequent nonadiabatic transition probability, $P_{\mathrm{cc}}$. Although the photodissociation of alkyl iodides involves multidimensional character, a stronger coupling strength may analogously result in a larger $P_{\mathrm{cc}}$. As the $\alpha$-branched alkyl group becomes heavier, the contribution from the bending fundamental and combination modes becomes larger relative to the CI stretch progression. 6,18,46 Accordingly, the umbrella mode of $\mathrm{CH}_{3}$ in the photodissociation of $\mathrm{CH}_{3} \mathrm{I}$ at 266 and $304 \mathrm{~nm}$ is found to reach $v_{2}=4$ in the I-product channel, ${ }^{22}$ whereas the $v_{2}$ mode of $\mathrm{C}_{2} \mathrm{H}_{5}$ can be extended to $>11$ and $>8$ in the photodissociation of $\mathrm{C}_{2} \mathrm{H}_{5} \mathrm{I}$ at 279.7 and $304 \mathrm{~nm}$, respectively. ${ }^{47}$ In this sense, the $\mathrm{C}_{2} \mathrm{H}_{5} \mathrm{I}$ photodissociation should tend to have a larger nonadiabatic transition rate that is, however, not clear enough in our previous work. $^{31}$

\section{Conclusion}

The photodissociation dynamics of ethyl iodide over the wavelength range of 294-308 $\mathrm{nm}$ has been investigated, in supplement to the work obtained similarly with shorter dissociation wavelengths. ${ }^{31}$ As expected, the I* channel, originating from the ${ }^{3} \mathrm{Q}_{0}$ state alone, is accompanied by colder internal states of $\mathrm{C}_{2} \mathrm{H}_{5}$, whereas the I channel, contributed by direct excitation and nonadiabatic transition, is with a hotter $\mathrm{C}_{2} \mathrm{H}_{5}$. The curve crossing probability rises rapidly around the conical intersection but remains almost constant after passing through the curve crossing. The ${ }^{1} \mathrm{Q}_{1}$ and ${ }^{3} \mathrm{Q}_{0}$ states in the exit region are thus expected to cross almost parallel along the dissociation coordinate. As compared to the photodissociation of $\mathrm{CH}_{3} \mathrm{I}$ at the same photolyzing wavelength, the nonadiabatic transition probabilities are slightly enhanced by an ethyl-substituted group.

Acknowledgment. This work is supported by National Taiwan University, the Ministry of Education, and the National Science Council of Taiwan, Republic of China under contract no. NSC 96-2113-M-002-027.

\section{References and Notes}

(1) Mulliken, R. S. J. Chem. Phys. 1940, 8, 382.

(2) Guo, H.; Schatz, G. C. J. Chem. Phys. 1990, 93, 393.

(3) Johnson, B. R.; Kittrell, C.; Kelly, P. B.; Kinsey, J. L. J. Phys. Chem. 1996, 100, 7743.

(4) Jung, Y. J.; Kim, Y. S.; Kang, W. K.; Jung, K. H. J. Chem. Phys. 1997, 107, 7187.

(5) Zhong, D.; Zewail, A. H. J. Phys. Chem. A 1998, 102, 4031.

(6) Samartzis, P. C.; Bakker, B. L.; Parker, D. H.; Kitsopoulos, T. N. J. Phys. Chem. A 1999, 103, 6106.

(7) Wu, G.; Jiang, B.; Ran, Q.; Zhang, J.; Harich, S. A.; Yang, X. J. Chem. Phys. 2004, 120, 2193.

(8) Eppink, A. T. J. B.; Parker, D. H. J. Chem. Phys. 1999, 110, 832.
(9) Xu, H.; Guo, Y.; Liu, S.; Ma, X.; Dai, D.; Sha, G. J. Chem. Phys. 2002, 117, 5722 .

(10) Rijs, A. M.; Janssen, M. H. M.; Chrysostom, E. T. H.; Hayden, C. C. Phys. Rev. Lett. 2004, 92, 123002.

(11) Hu, C.; Pei, S.; Chen, Y. L.; Liu, K. J. Phys. Chem. A 2007, 111, 6813

(12) Eppink, A. T. J. B.; Parker, D. H. J. Chem. Phys. 1998, 109, 4758. (13) van Veen, G. N. A.; Baller, T.; de Vires, A. E.; van Veen, N. J. A. Chem. Phys. 1984, 87, 405.

(14) Amatatsu, Y.; Yabushita, S.; Morokuma, K. J. Chem. Phys. 1996, 104, 9783.

(15) Talukdar, R. K.; Vaghjiani, G. L.; Ravishankara, A. R. J. Chem. Phys. 1992, 96, 8194.

(16) Alekseyev, A. B.; Liebermann, H.-P.; Buenker, R. J.; Yurchenko, S. N. J. Chem. Phys. 2007, 126, 234102.

(17) de Nalda, R.; Lzquierdo, J. G.; Dura, J.; Banares, L. J. Chem. Phys. 2007, 126, 021101.

(18) Philips, D. L.; Lawrence, B. A.; J, J.; Valentini, J. Phys. Chem. 1991, 95, 9085.

(19) Philips, D. L.; Myers, A. B.; Valentini, J. J. J. Phys. Chem. 1992, 96, 2039.

(20) Philips, D. L.; Meyers, A. B. J. Chem. Phys. 1991, 95, 226.

(21) Paterson, C.; Codwin, F. G.; Gorry, P. A. Mol. Phys. 1987, 60, 729 .

(22) Li, G.; Shin, Y. K.; Hwang, H. J. J. Phys. Chem. A 2005, 109, 9226

(23) Uma, S.; Das, P. K. J. Chem. Phys. 1996, 104, 4470.

(24) Kim, Y. S.; Kang, W. K.; Kim, D. C.; Jung, K. H. J. Phys. Chem. A 1997, 101, 7576.

(25) Kang, W. K.; Jung, K. W.; Kim, D. C.; Jung, K. H.; Im, H. S. Chem. Phys. 1995, 196, 363.

(26) North, S. W.; Sears, T. J.; Hall, G. E.; Suzuki, T. Chem. Phys. 1996, 211,515 .

(27) Rattigan, O. V.; Shallcross, D. E.; Cox, R. A. J. Chem. Soc., Faraday Trans. 1997, 93, 2839.

(28) Roehl, C. M.; Burkholder, J. B.; Moortgat, G. K.; Ravishankara,

A. R.; Crutzen, P. J. J. Geophys. Res. 1997, 102, 12819.

(29) Brewer, P.; Das, P.; Ondrey, G. S. J. Chem. Phys. 1983, 79, 720.

(30) Godwin, F. G.; Gorry, P. A.; Hughes, P. M. Chem. Phys. Lett. 1987, $135,163$.

(31) Tang, Y.; Lee, W. B.; Hu, Z.; Zhang, B.; Lin, K. C. J. Chem. Phys. 2007, 126, 064302 .

(32) Tang, Y.; Lee, W. B.; Zhang, B.; Lin, K. C. J. Phys. Chem. A. 2008, 112, 1421.

(33) Hu, Z.; Lee, W. B.; Zhang, X. P.; Wei, P. Y.; Lin, K. C. ChemPhysChem. 2008, 9, 422.

(34) Fan, H.; Pratt, S. T. J. Chem. Phys. 2005, 123, 204301.

(35) Lee, K. S.; Lim, J. S.; Ahn, D. S.; Choi, K. W.; Kim, S. K.; Choi,

Y. S. J. Chem. Phys. 2006, 124, 124307.

(36) Zare, R. N.; Herschbach, D. R. Proc. IEEE. 1963, 51, 173.

(37) Zare, R. N. Mol. Photochem. 1972, 4, 1.

(38) Larsen, J. J.; Sakai, H.; Safvan, C. P.; Wendt-Larsen, I.; Stapelfeldt, H. J. Chem. Phys. 1999, 111, 7774.

(39) Sugita, A.; Mashino, M.; Kawasaki, M.; Matsumi, Y.; Gordon, R. J.; Bersohn, R. J. Chem. Phys. 2000, 112, 2164.

(40) Kaziannis, S.; Kosmidis, S. C. Chem. Phys. Lett. 2005, 401, 115.

(41) Janssen, M. H. M.; Mastenbroek, J. W. G.; Stolte, S. J. Phys. Chem.

A 1997, 101, 7605 .

(42) Gedanken, A. Chem. Phys. Lett. 1987, 137, 462.

(43) Lao, K. Q.; Person, M. D.; Xayariboun, P.; Butler, L. J. J. Chem. Phys. 1990, 92, 823 .

(44) Ajitha, D.; Wierzbowska, M.; Lindh, R.; Malmqvist, P. A. J. Chem. Phys. 2004, 121, 5761.

(45) Codwin, F. G.; Paterson, C.; Gorry, P. A. Mol. Phys. 1987, 61, 827.

(46) Knoblauch, N.; Strobel, A.; Fischer, I.; Bondybey, V. E. J. Chem. Phys. 1995, 103, 5417.

(47) Bi, W. B.; Xu, X. L.; Huang, J. G.; Xiao, D. Q.; Zhu, Q. H. Sci. China Ser. B-Chem. 2007, 50, 476.

JP805118W 